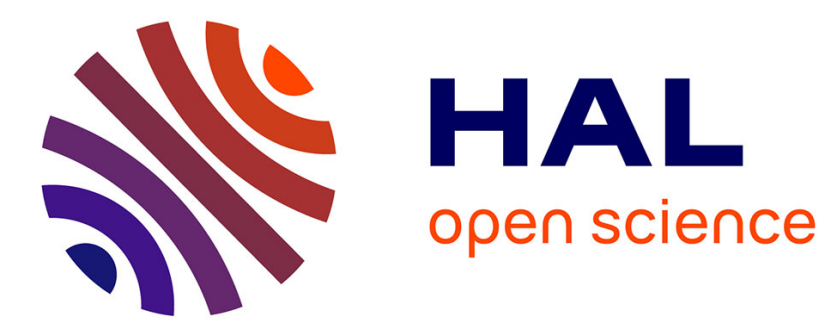

\title{
Determination of the Streamers Characteristics Propagating in Liquids using the Electrical Network Computation
}

\author{
Thomas Aka-Ngnui, Abderrahmane Beroual
}

\section{- To cite this version:}

Thomas Aka-Ngnui, Abderrahmane Beroual. Determination of the Streamers Characteristics Propagating in Liquids using the Electrical Network Computation. IEEE Transactions on Dielectrics and Electrical Insulation, 2006, 13 (3), pp.572-579. hal-00135194

\author{
HAL Id: hal-00135194 \\ https://hal.science/hal-00135194
}

Submitted on 7 Mar 2007

HAL is a multi-disciplinary open access archive for the deposit and dissemination of scientific research documents, whether they are published or not. The documents may come from teaching and research institutions in France or abroad, or from public or private research centers.
L'archive ouverte pluridisciplinaire HAL, est destinée au dépôt et à la diffusion de documents scientifiques de niveau recherche, publiés ou non, émanant des établissements d'enseignement et de recherche français ou étrangers, des laboratoires publics ou privés. 


\title{
Determination of the Streamers Characteristics Propagating in Liquids using the Electrical Network Computation
}

\author{
T. Aka-Ngnui and A. Beroual \\ Ecole Centrale de Lyon, CE.G.E.LY. C.N.R.S. U.M.R. 5005 \\ 36, Avenue Guy De Collongue \\ 69134 Ecully Cedex, France
}

\begin{abstract}
This work is devoted to the modeling of branching streamers propagating in transformer oil using an equivalent electrical network and the electrical network computation. The proposed model enables to determine the different characteristics of the streamer (i.e., the associated current and the electrical charge, the power and the energy injected in the liquid, the local electric field at the streamer head, the streamer shape and its velocity, the mobility of the charge carriers within the streamer channels, the local viscosity and temperature). It's shown through the simulated values of the mobility of charge carriers, the local viscosity and temperature that both electronic and gaseous mechanisms are implicated in the streamer development. The gaseous nature of streamers and the role of the local electric field are evidenced. The influence of the conductivity and additives as well as the electrode gap on the propagation velocity of positive streamers is analyzed.
\end{abstract}

Index Terms - Oil insulation, streamer propagating, electrical network computation.

\section{INTRODUCTION}

THE mechanisms involved in the pre-breakdown and breakdown phenomena in transformer oil have been the subject of many studies during the last decades. Our understanding has been greatly improved thanks to the use of fast optical detecting systems and measuring new techniques, which allow us to follow the different stages leading to breakdown.

It is well known that the main parameters governing the streamer propagation through the electrode gap and its characteristics are the local electric field at the streamer tip, the average electric field in the gap and the physicochemical properties of the medium [1]. On the other hand, according to numerous data reported in the literature, there is a close correlation between the streamer shape and its propagation velocity, the associated current shape and emitted light in many liquids [2,3]. It would be then useful, thanks to this knowledge, to elaborate an appropriate model enabling to predict the streamer characteristics in order to reduce the time and the costs of long and often hard experiments.

In previous papers, we have shown the utility of the equivalent electrical network for the modeling of discharges and the elaboration of predictive models [4 - 6]. This paper is aimed at the modeling of the multi-channel streamers propagating within a dielectric liquid submitted to a divergent electric field (i.e., in a

Manuscript received on 15 September 2005, in final form 6 February 2006. point-plane electrode arrangement) using an equivalent electrical network, physical laws and energetic considerations. We first present briefly the bases of the model. Then, we give some results of simulations such as the streamer shape, the current and electrical charge, the local electric field, the propagation velocity in oil, the mobility of charge carriers, the local viscosity and temperature.

We analyze especially the influence of the streamer conductivity, the electrode gap and the additives on these characteristics of streamers. Then, we discuss the simulated results in the light of the experimental results known in literature, the physical nature of the streamers and the mechanisms responsible of their development.

\section{ALGORITHM OF STREAMER GENERATION}

The algorithm that we use is that described in a previous work [4]. We remind here the main features of our model. It consists in simulating the propagation of multi-channel streamers mainly basing on an electrical network method. It uses two criteria for the propagation of the streamers: (i) the electric field at the head of each generated channel of the streamer, $E_{\text {head }}$, which must be higher than a threshold value $E_{\text {threshold }}$ required for the initiation of one of the different types of streamers [1] ( $E_{\text {head, }}$ can be calculated using the hyperboloidal approximation); and (ii) the energy $W_{\text {joule }}$ 
supplied by the electric field $E_{\text {head }}$ must be higher than the latent heat of vaporization of the liquid, $L_{v}$

$W_{\text {joule }}=R_{\text {est }} I_{R}^{2} \Delta t$

where $R_{\text {est }}$ is the streamer resistance, $I_{R}$ is the resistive current and $\Delta t$ the step time.

At each time and for a given streamer branch, if both propagation criteria are satisfied, then we calculate the length and direction of the new branch. Thereafter we achieve a temporal recursive resolution on this algorithm while deducting at each time all physical parameters [4].

\section{EQUIVALENT ELECTRICAL ELEMENTS}

The equivalent electrical elements have been described in previous work [4]. Each streamer branch is assumed to a cylindrical channel of conductivity $\sigma$, length $l$ and radius $r_{0}$; its resistance $R_{\text {est }}$ is then

$R_{e s t}=l / \sigma \pi r_{0}^{2}$

Two types of channels are considered: the "free channels" and "tied channels". The free channels are the channels having one extremity no connected to other channels and the tied channels are the channels that have their both extremities connected to at least a channel. To each free channel, we associate a capacitor $C_{\text {capa }}$ constituted of the free extremity of the channel and the opposite electrode. This capacitor is calculated using a hyperboloidal approximation and by taking into account the solid angle constituted by the streamer head (i.e., the free extremity of a free channel) and having the electrode plane as a base.

The structure so generated and the above hypotheses enable to build an electric circuit that we solve using the computation electrical network method. Thus we determine the current and the voltage.

\section{NODAL RESOLUTION}

At each time, we compute the physical parameters characterizing the site at which a new channel will be generated, i.e., the current and the electrical charge, the energy and the power injected in the insulating medium as well as the streamer velocity, the local electrical field at the streamer head, the local viscosity $(v)$ and temperature $(T)$, and the mobility $(\mu)$ of charge carriers within the generated channel [4]. The relationships we used to calculate $v, T$ and $\mu$ are those given elsewhere $[4,7]$. From the topology of this system, we establish the equations of the electrical network that we then solve by a nodal method. The nodal resolution consists in constructing for each streamer jump, the branch-node incidence matrix $\mathbf{A}$, the primitive admittance matrix $\mathbf{Y}$ and the voltage and current source matrixes $\mathbf{E}$ and $\mathbf{I}$ [4]. Thereafter, we compute the nodal voltages, the voltage at the branches and the currents within the branches.

\section{INFLUENCE OF THE STREAMER 5.1 CONDUCTIVITY}

To validate our model, consider a point-plane electrode arrangement with a gap of $10 \mathrm{~mm}$, immersed in a dielectric liquid of permittivity $\mathcal{E}_{r}=2$ and submitted to a dc voltage of $30 \mathrm{kV}$; the radius of the point electrode being $R_{p}=5 \mu \mathrm{m}$. Figure 1 gives examples of streamer patterns obtained for two values of the streamer conductivity [8], namely $\sigma=5 \Omega^{-1} \cdot \mathrm{m}^{-1}$ and $\sigma=$ $50 \Omega^{-1} \cdot \mathrm{m}^{-1}$, and a radius of channels $r_{0}=5 \mu \mathrm{m}$. These are similar to those observed experimentally $[1,4]$. We remark that the more conducting the streamer, the larger is its final length (i.e., faster).

Now let analyze the physical parameters associated to these types of streamer.

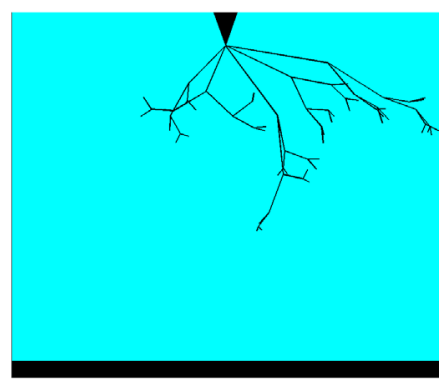

(a)

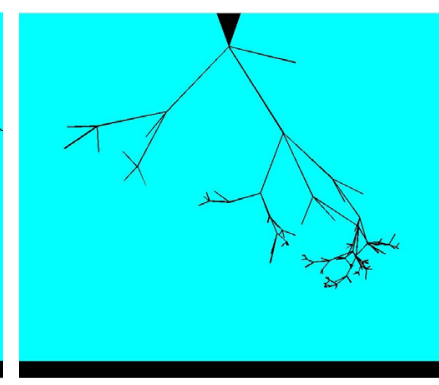

(b)
Figure 1. Example of streamer patterns obtained for a point-plane arrangement under dc voltage after a time simulation of $30 \mu \mathrm{s}$; point radius $R p=5 \mu \mathrm{m}$; electrode gap $D=10 \mathrm{~mm}$; applied voltage $U_{0}=$ $30 \mathrm{kV}$ : (a) $\sigma=5(\Omega \mathrm{m})^{-1}$ and (b) $\sigma=50(\Omega \mathrm{m})^{-1}$.

\subsection{CURRENT AND INJECTED CHARGE}

Each time when a channel (streamer branch) is generated, we compute the instantaneous injected charge (i.e., the elementary charge) and the accumulated injected charge (i.e., the total charge) (Figure 2).

We observe that the streamer charge consists of steps the amplitudes of which are of about several tens of picocoulombs as those observed experimentally for streamers of such patterns $[3,9]$. The streamer charge increases with its conductivity; in the same way, the duration between steps is also reduced leading to a more continuous propagation and then to the increase of the propagation velocity. Note that the simulated charges do not concern only the main streamer branch (i.e., the largest branch).

\subsection{ELECTRIC FIELD}

Figure 3 gives the variation of the local electrical field at the free channel head, computed using the hyperboloidal approximation. We note that the values of the local electric field are high enough to initiate primary and secondary streamers [1]; they vary between 5 and $15 \mathrm{MV} / \mathrm{cm}$. For voltages higher than $30 \mathrm{kV}$, other types of streamers can be initiated. 

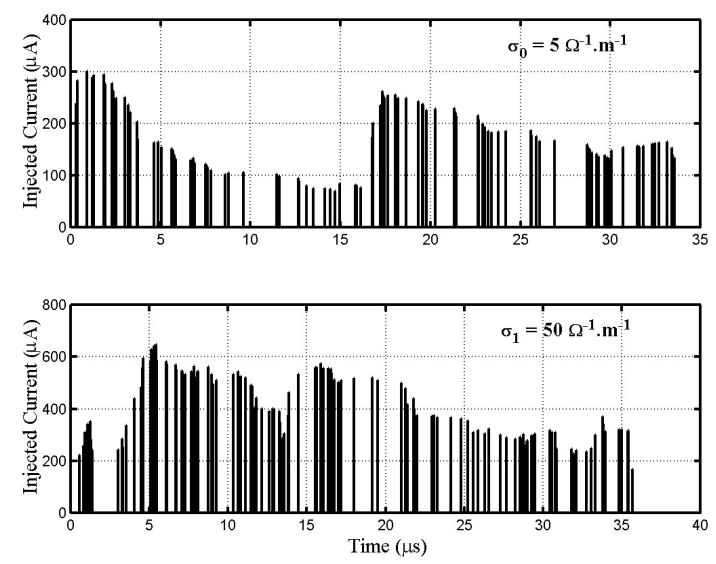

(a)

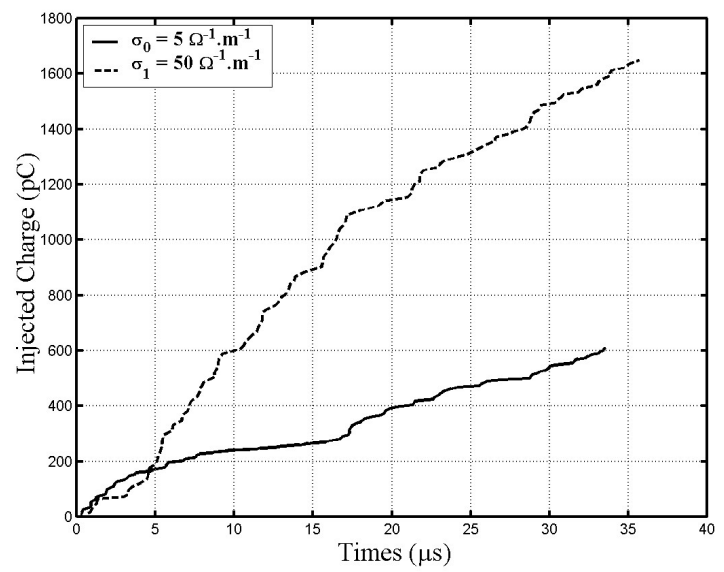

(b)

Figure 2. Injected (a) currents and (b) electrical charge associated to the simulated streamer of Figure 1.

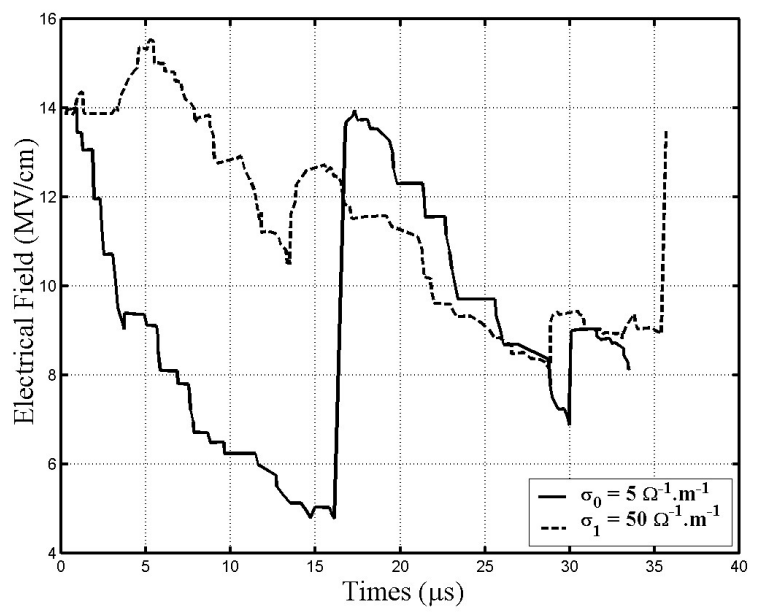

Figure 3. Electric field at the head of the simulated streamer of Figure 1.

\subsection{STREAMER VELOCITY}

The instantaneous streamer velocity is calculated using the relationship established elsewhere [3] $v=\left[2 \beta P_{s c e} / \rho_{s} \pi r_{0}^{2}\right]^{1 / 3}$

$\beta$ is the available part of the energy used for the streamer displacement and $P_{s c e}$ is the instantaneous power injected into the medium. The analysis of different experimental results for branching streamers, shows that $\beta$ is between 10 and $20 \%$ of the total energy injected in the medium [3]; in our simulations we set $\beta=0.10$.

Figure 4 gives the variation of the simulated instantaneous velocity for the same test conditions.

We observe that the values of the instantaneous velocities are of some $\mathrm{km} / \mathrm{s}$ (see Figure 4). Note that the corresponding average velocities are smaller than that of the instantaneous ones due to the duration of the pause time between two partial displacements, which is large enough compared to the effective propagation time of a partial displacement. These velocities are in the same range as those reported in the literature for this type of streamers [1]. On the other hand, the variation of the velocity follows that of the electric field indicating that the local electric field plays also an important role not only in the generation of the streamer type but also in the mode of propagation.

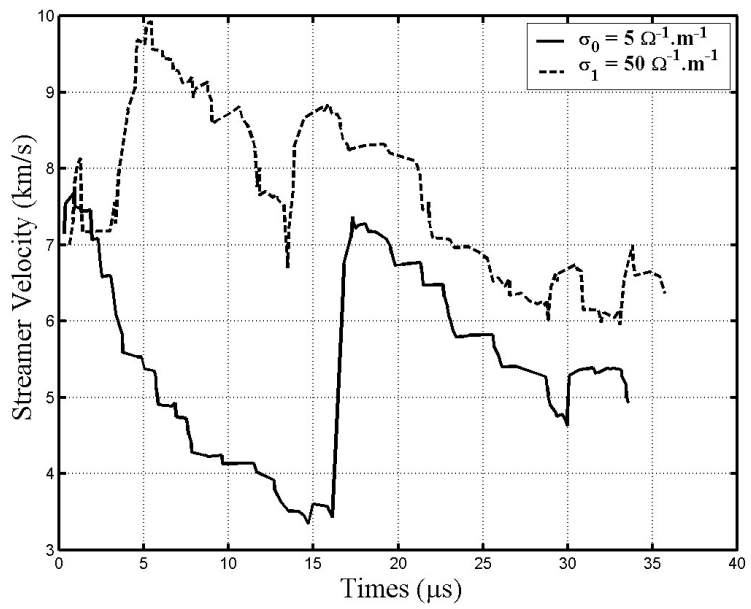

Figure 4. Instantaneous velocity of the simulated streamer of Figure 1.

\subsection{LOCAL VISCOSITY AND TEMPERATURE}

The local viscosity, $\eta_{v}$, of the matter (saturated vapor) constituting the streamer channels generated at each time, can be calculated using our model and Walden's law (extended to saturated vapor) [7] even if this latter is questionable for very high electric fields

$K_{m} \eta_{v}=$ cste

The obtained values for $\eta_{v}$ are of some $10^{-3} \mathrm{cp}$ (Figure 5); these are close to those corresponding to the gases. Let us remind that, at the ambient, the viscosity of air, and oils are respectively $15 \times 10^{-3} \mathrm{cp}$ and 150 to $800 \mathrm{cp}$. Therefore, these simulations confirm the gaseous nature of the streamers channels $[1,2]$. 


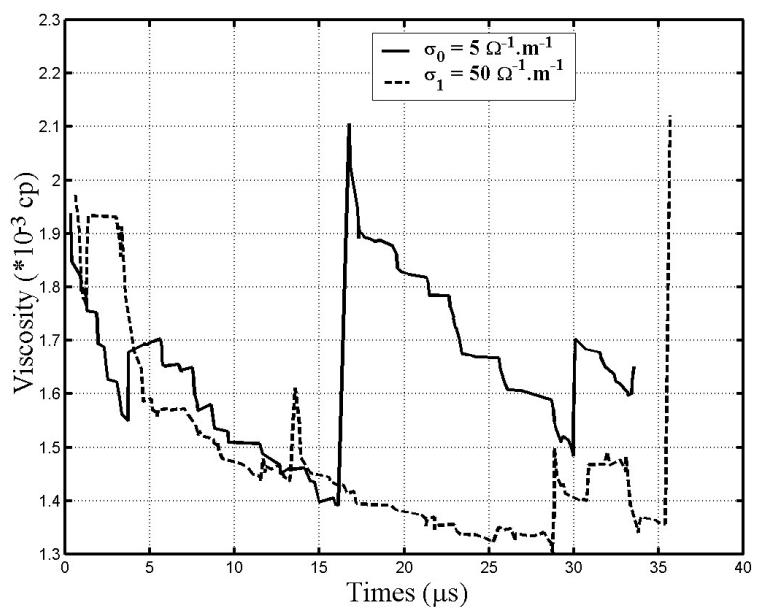

Figure 5. Local viscosity of the streamer channels of Figure 1.

The temperature, $T$, within the channels can be deduced from the relationship between the viscosity $\eta_{v}$ and the activation energy $W_{\text {act }}$ assumed to be constant [7]

$T=W_{\text {act }} / k \log \left(\eta_{v} /\right.$ cste $\left._{T}\right)$

where $k$ is the Boltzmann constant and cste $_{T}$ a constant.

Figure 6 gives the variation of temperature within the streamer channels. We observe that these temperatures vary between 380 and $430 \mathrm{~K}$ (or 107 and $157^{\circ} \mathrm{C}$ ) and they are of the same range as those required for the vaporization of most of dielectric liquids.

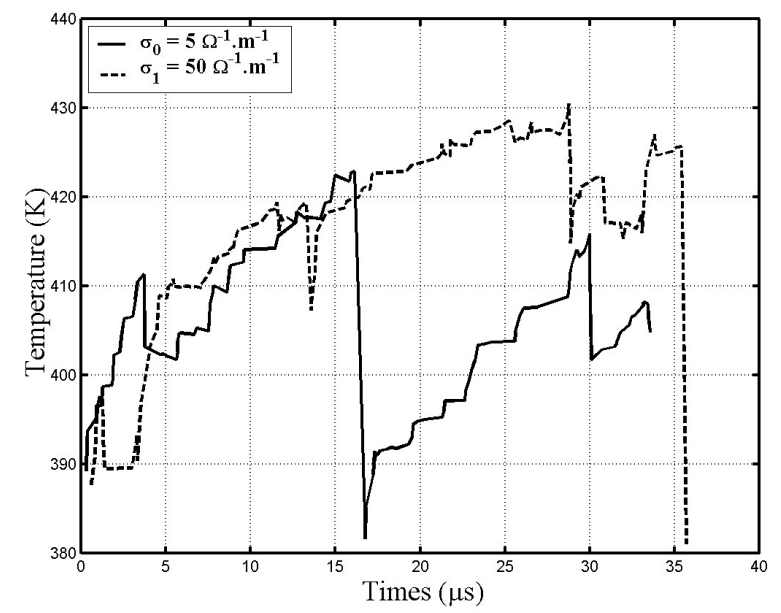

Figure 6. Local temperature within the streamer channels of Figure 1.

\section{INFLUENCE OF THE ELECTRODE GAP}

Besides the physicochemical properties of the liquid, the shape, the velocity and the propagation mode of streamers depend enormously on the electric field which in turn is a function of the applied voltage, the electrode geometry and the space charge emanating from the liquid bulk or injected.

In the following, we present the results of simulations relating to the influence of the electrode gap, $D$, and the voltage, $U$, on the shape and the velocity of streamers, the electrical charge and the local electrical field in a point-plane electrode arrangement immersed in a dielectric liquid of permittivity $\varepsilon_{r}=2$ and submitted to a dc voltage; the radius of the point electrode being $R_{p}$.

\subsection{SHAPE AND VELOCITY OF STREAMERS}

Figures 7 and 8 give respectively examples of streamer patterns and the variation of the simulated instantaneous velocity for different electrode gaps, submitted to a dc voltage of $30 \mathrm{kV}$, after $1.4 \mu \mathrm{s}$ of propagation. We use some experimental characteristics of branching streamers that we get previously [6] namely a conductivity $\sigma=5 \Omega^{-1} \cdot \mathrm{m}^{-1}$ and a radius of channels $r_{0}=5 \mu \mathrm{m}$.

We remark that the streamer shape are similar to those observed experimentally. Note that different streamer patterns can be obtained for the same conditions (i.e, for the same geometry and applied voltage).

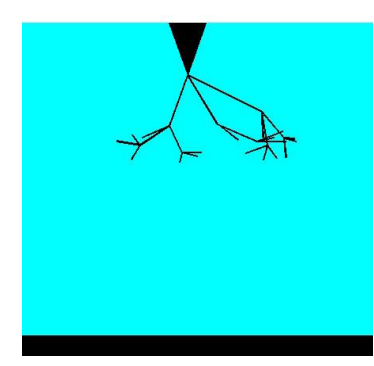

(a)

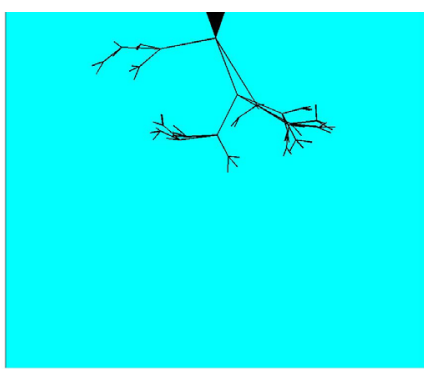

(c)

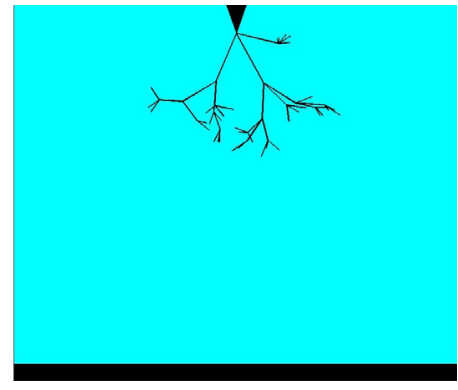

(b)

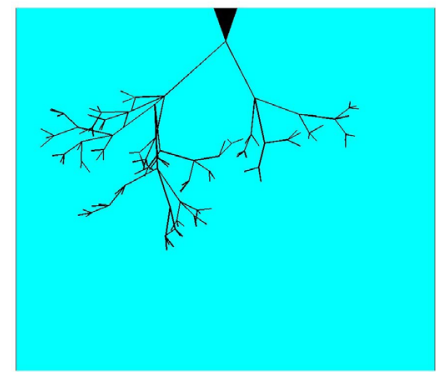

(d)
Figure 7. Examples of streamer patterns obtained for a point-plane electrode arrangement under dc voltage after a time simulation of 1.4 $\mu \mathrm{s} ; D=5(a), 10(b), 15(c)$ and $35(d) \mathrm{mm} ; R_{p}=3 \mu \mathrm{m}, U=30 \mathrm{kV}$.

The simulated values of velocities (some $\mathrm{km} / \mathrm{s}$ ) also are of the same range as those reported in the literature for this type of streamers $[1,3]$. On the other hand, for the same voltage and radius of electrode point, the velocity at the beginning of propagation is higher when the electrode gap is smaller. Note that these results are obtained by assuming the conductivity of the streamers channels constant while it varies depending on the experimental conditions namely the electrode gap here. Indeed, the higher the conductivity of streamer channels, the higher the propagation velocity is. Figure 4 gives an example of the variation of the velocity for two conductivities of streamer channels. 


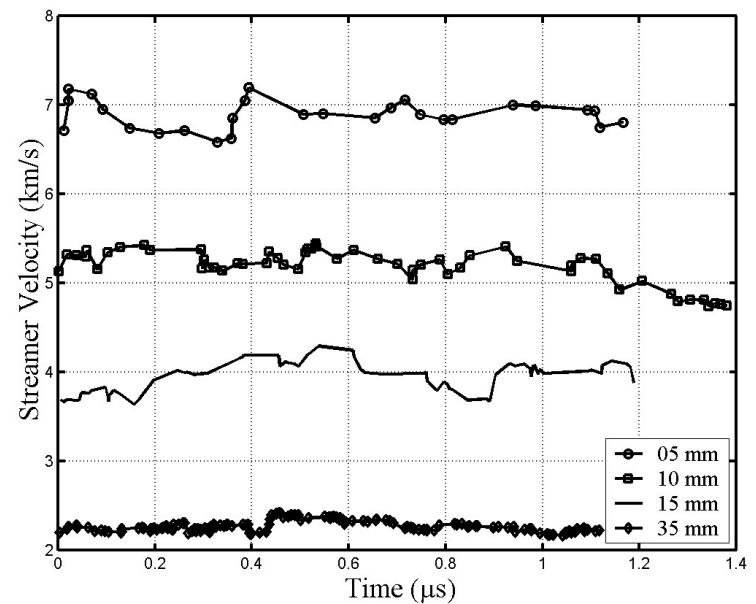

Figure 8. Streamer velocity for $D=5,10,15$ and $35 \mathrm{~mm} ; R_{p}=3 \mu \mathrm{m}$, $U=30 \mathrm{kV}, \sigma=5 \Omega^{-1} \cdot \mathrm{m}^{-1}$.

\subsection{ELECTRIC FIELD}

The variation of the velocity follows that of the electric field (Figure 9) indicating that the local electric field plays also an important role not only in the generation of the streamer type but also in the mode of propagation.

According to the values of this field, it is possible to initiate different structures of streamers $[1,4,10]$. The local electrical field obtained by simulation is in the range $10-15 \mathrm{MV} / \mathrm{cm}$. Such values are quite in good accordance with that estimated experimentally $[3,10]$.

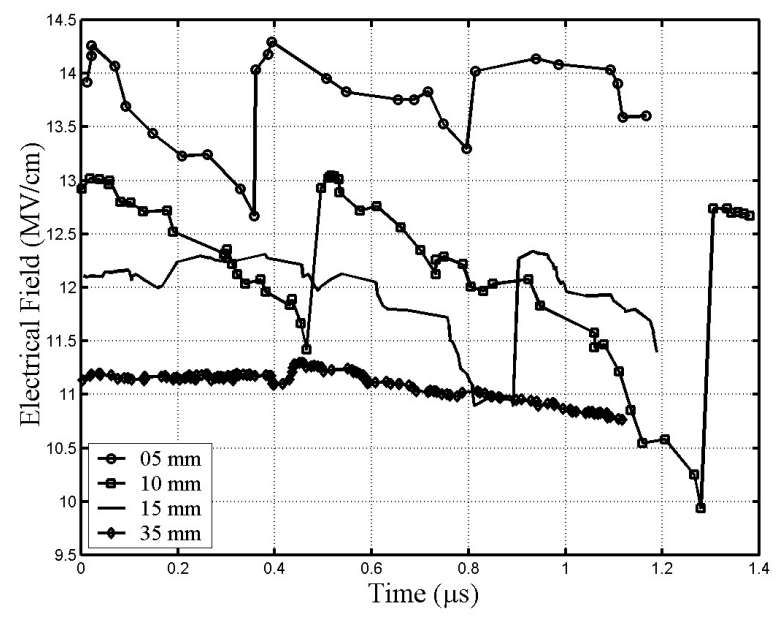

Figure 9: Electrical field at the head of simulated streamers of figure 7: for $D=5,10,15$ and $35 \mathrm{~mm} ; U=30 \mathrm{kV}, \sigma=5 \Omega^{-1} \cdot \mathrm{m}^{-1}, R_{p}=3$ $\mu \mathrm{m}$.

\subsection{INJECTED CHARGE}

The electric charges are obtained by cumulative integral on the whole of simulation. The values we obtained vary by steps from some $\mathrm{pC}$ to some hundreds of $\mathrm{pC}$ (Figure 10). They are of the same range as that measured experimentally for the same test parameters.

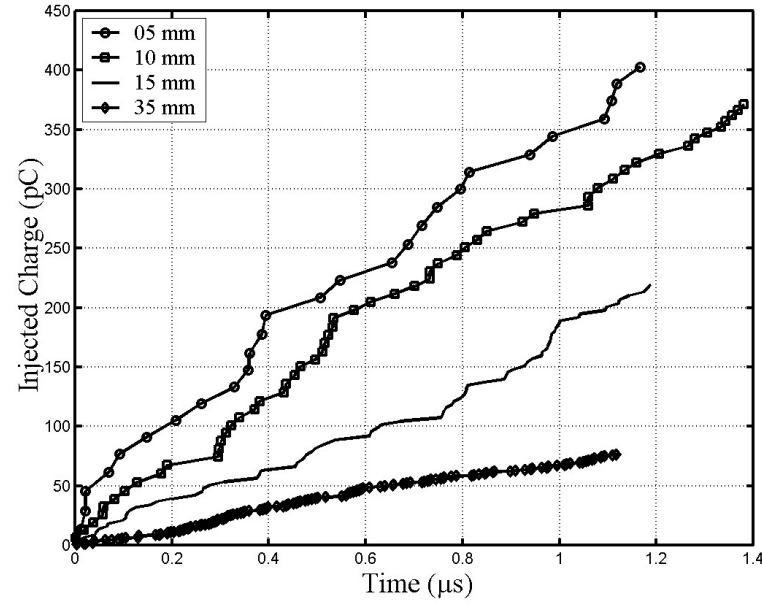

Figure 10. Injected electrical charge for $D=5,10,15$ and $35 \mathrm{~mm}$; $R_{p}=3 \mu \mathrm{m}, U=30 \mathrm{kV}, \sigma=5 \Omega^{-1} . \mathrm{m}^{-1}$.

We observe that the electrical charge increases when the electrode gap decreases, indicating the influence of capacitive effects of the electrode arrangement. At $1 \mu \mathrm{s}$, the streamer charge accumulated for $5 \mathrm{~mm}$ is roughly twice more important than that of $15 \mathrm{~mm}$ and five times that of $35 \mathrm{~mm}$. This explains the high values of electric fields and velocities for small gaps.

\section{ACTION OF ADDITIVES}

The action of additives is simulated by a density of surface charge at the active head of streamer such as

$Q_{s c}(t)=\alpha \cdot \exp (-t / \delta)$

where $\alpha$ is the initial added charge corresponding to the quantities of additives and $\delta=R C(R$ streamer resistance and $C$ capacitor constituted of the free extremity of the channel and the opposite plane electrode). This exponential decrease represents the re-combination of charge carriers. The contribution of the space charge resulting from additives, to the electrical field $E_{\text {head }}$, is given by

$E_{s c}=Q_{s c} / \varepsilon_{2} S \quad$ where $\quad S=2 \pi r_{o}^{2}$.

In the following, we present the results of simulations relating to the influence of electrode gap, $D$, and the voltage, $U$, on the shape and velocity of positive streamers, the electrical charge and the local electrical field in a point-plane electrode arrangement immersed in transformer oil of dielectric constant $\varepsilon_{r}=2.2$ and submitted to a dc voltage, the radius of the point electrode being $R_{p}$.

\subsection{INJECTED CURRENTS AND CHARGES}

Figures 11 and 12 give respectively an example of a streamer pattern and the currents accompanying streamers 
propagating in transformer oil alone and in presence of positive or negative space charge (SC) resulting of additives, submitted to a dc voltage of $30 \mathrm{kV}$. The positive and negative charge carriers can result, for instance, of the adjunction of low ionization potential or electronic scavenger compounds.

The simulation of propagation of positive streamers (Figure 11) without additives lasted only $80 \mu$ s (Figure 12a) while with a contribution of positive charge carriers, the duration of the propagation is reduced to $50 \mu s$ (Figure 12b) explaining the reinforcement of the electrical field at the head of the active streamer supporting more the propagation contrary to the addition of negative charge carriers which lasted $300 \mu \mathrm{s}$ (Figure 12c) showing the reduction of the electric field at the head of the streamer.

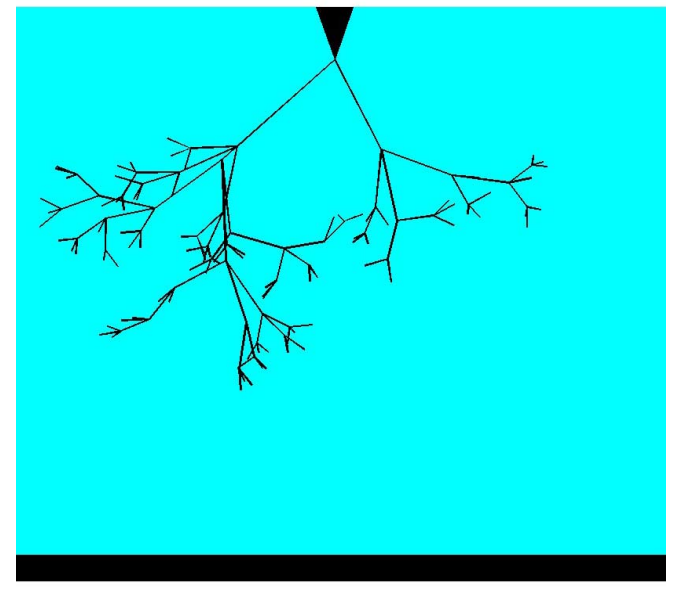

Figure 11: Example of streamer patterns obtained for a point-plane electrode arrangement under dc voltage: $D=10 \mathrm{~mm} ; R_{p}=3 \mu \mathrm{m} ; U$ $=+30 \mathrm{kV}$.

We use some experimental characteristics of branching streamers that we get previously [4] namely a conductivity $\sigma=$ $5 \Omega^{-1} \cdot \mathrm{m}^{-1}$ and a radius of channels $r_{0}=5 \mu \mathrm{m}$.

We remark that the streamer shape is similar to those observed experimentally. Note that different streamer patterns can be obtained for the same conditions (i.e, for the same geometry and voltage).

They are of the same range as that measured experimentally for the same test conditions. We observe that the obtained cumulative capacitive charges at the streamer head when the point is positive confirm our waiting [10-11]. There is an increase in the charge with a contribution of positive charge carriers and a reduction after an addition of negative charge carriers.

\subsection{ELECTRICAL FIELD AND STREAMER VELOCITY}

Figure 14 seems to contradict the above explanations. Indeed the three simulations having generated the same tree structure (Figure 11) out of three different lapses of time (80, 50 and $300 \mu \mathrm{s}$ ), at a given time, the active head of streamer at which the electric field is calculated is not the same one for the three simulations. Thus the most adapted comparison of the electric fields is that of the average fields on all simulation.

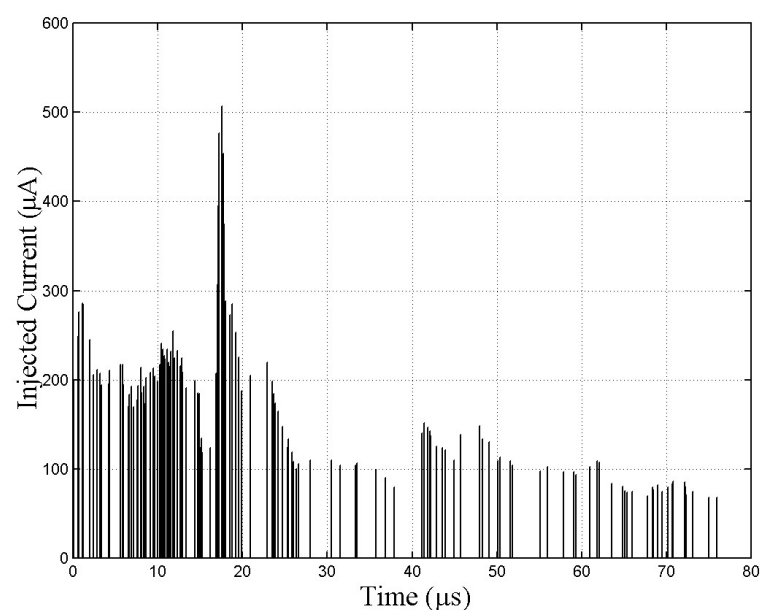

(a)

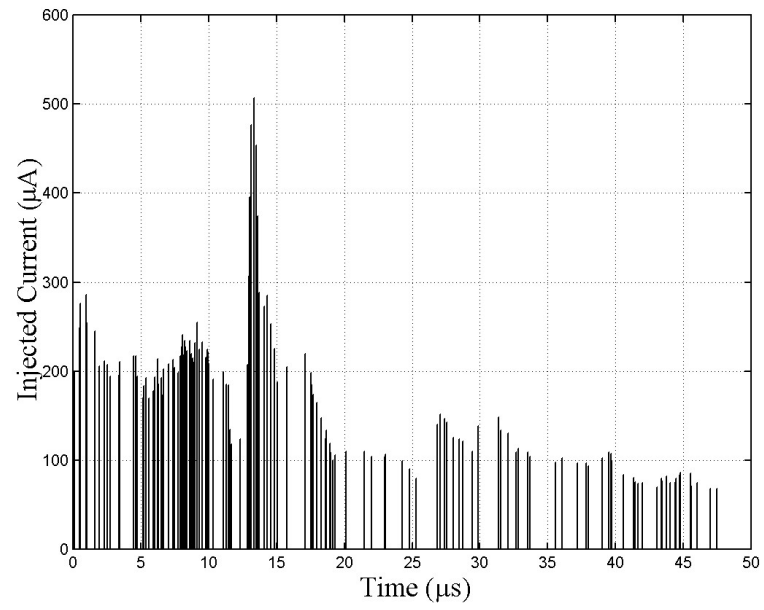

(b)

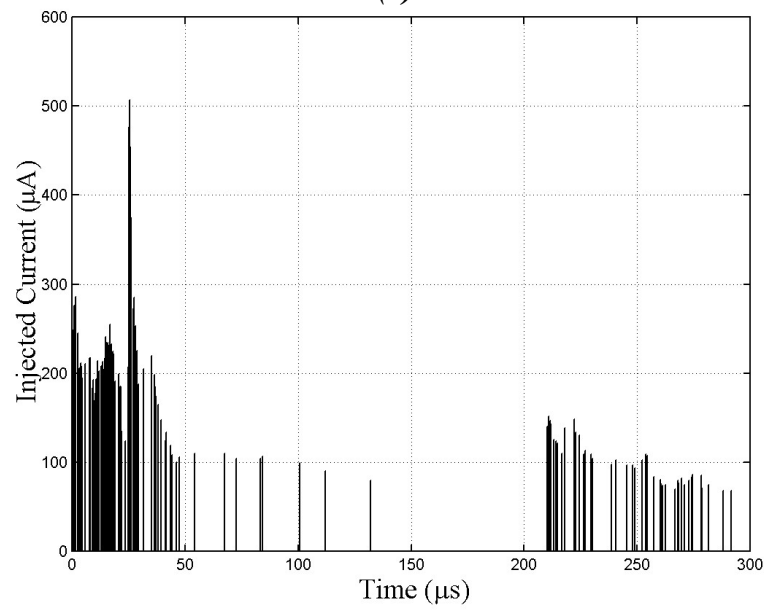

(c)

Figure 12. Injected currents in transformer oil (a) without space charge (no SC); (b) with addition of positive charge carriers (SC positive); (c) with addition of negative charge carriers (SC negative): $D=10 \mathrm{~mm} ; R_{p}=3 \mu \mathrm{m} ; U=+30 \mathrm{kV}$.

The average electric fields are respectively $7.86 \mathrm{MV} / \mathrm{cm}$, $11.06 \mathrm{MV} / \mathrm{cm}$ and $4.90 \mathrm{MV} / \mathrm{cm}$ for oil without space charge, with a positive and a negative charge carrier. 
As concern the velocities (Figure 15), we obtain a mean velocity identical, in the three simulations, equal to $4.07 \mathrm{~km} / \mathrm{s}$ showing the weak effect of additives on the velocity of positive streamers $(+30 \mathrm{kV})$.

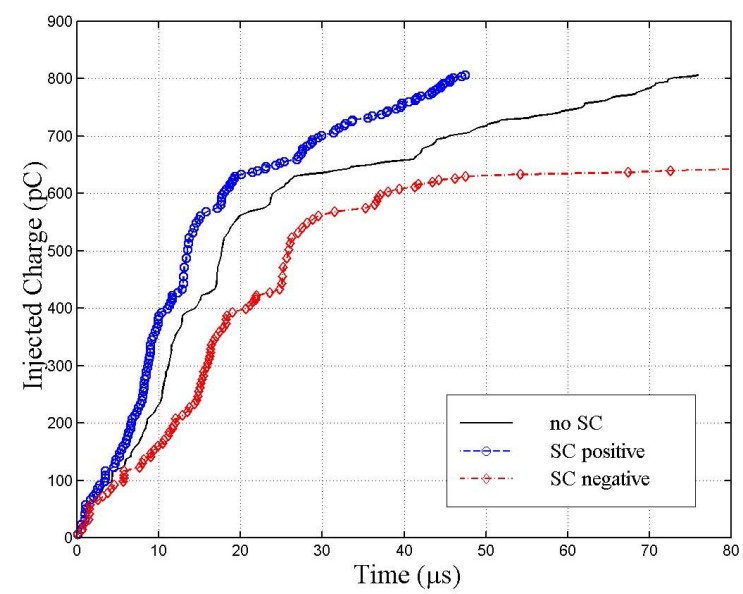

Figure 13. Injected electrical charge for $D=10 \mathrm{~mm} ; R p=3 \mu \mathrm{m} ; U$ $=30 \mathrm{kV}$

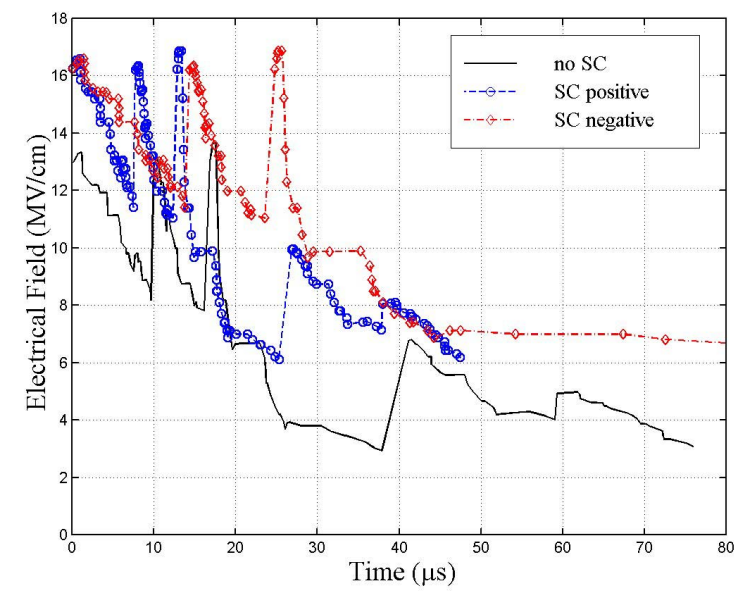

Figure 14. Electrical field for $D=10 \mathrm{~mm} ; R_{p}=3 \mu \mathrm{m} ; U=+30 \mathrm{kV}$.

\subsection{VELOCITY AND TEMPERATURE}

In the same way, the model enables to compute the local viscosity of the streamer channels and temperature within the branches (Figures 16 and 17) [4].

The values obtained, for the local viscosity of the matter constituting the streamer channels generated, are of some $10^{-3}$ $\mathrm{cp}$; these are close to those corresponding to the gases [12]. Therefore, these simulations confirm the gaseous nature (saturated vapor) of the streamer channels. We also observe that the temperatures vary between 300 and $500 \mathrm{~K}$ and they are of the same range, as those required for the vaporization of the most dielectric liquids.

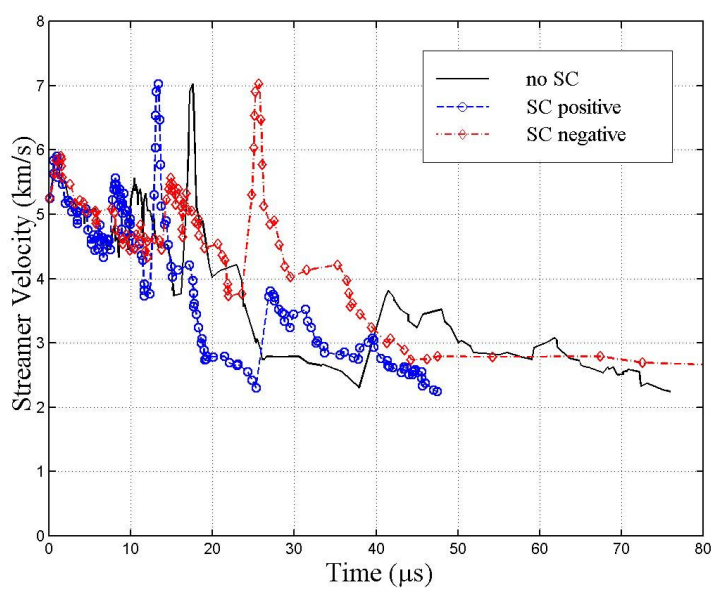

Figure 15. Streamer velocity for $D=10 \mathrm{~mm} ; R_{p}=3 \mu \mathrm{m}, U=30 \mathrm{kV}$.

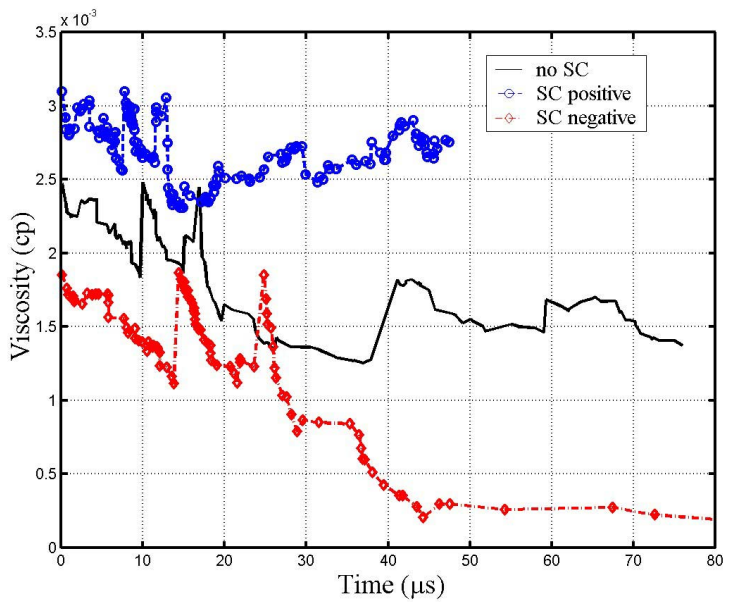

Figure 16. Local viscosity of the streamer channels.

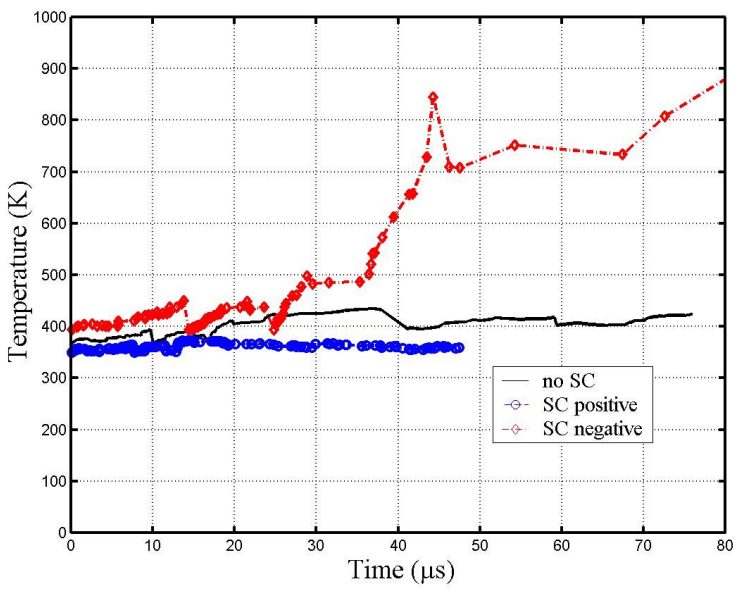

Figure 17. Local temperature within the streamer channels.

\section{CONCLUSION}

The use of equivalent electrical network appears as an helpful tool to model the multi-channel streamers propagating within a dielectric liquid and enabling to determine the different characteristics of the streamer (i.e., the associated current and the electrical charge, the power and the energy 
injected in the liquid, the local electric field at the streamer head, the streamer shape and its velocity, the mobility of the charge carriers within the streamer channels, the local viscosity and temperature).

These simulated results are quite in a good accordance with those obtained experimentally and reported in the literature. Our modeling shown that the streamers propagation depend not only on the test conditions but also on the local electric field. The local electric field at different successive steps of streamer propagation, which in its turn depends on the elementary charges at the streamer head, imposes the further stages of propagation, i.e., the streamer pattern and its propagation mode. The addition of positive or negative charge carriers has not a significant effect on the propagation velocity of positive streamers. The simulated values of the mobility of charge carriers, the local viscosity and temperature show that both electronic and gaseous mechanisms are implicated in the streamer development.

\section{REFERENCES}

[1] A. Beroual, M. Zahn, A. Badent, K. Kist, A.J. Schwabe, H. Yamashita, K. Yamazawa, M. Danikas, W.G. Chadband and Y. Torshin, Liquid Dielectric Committee International Study Group - IEEE DEIS: "Propagation and Structure of Streamers in Liquid Dielectrics", IEEE Electr. Insul. Magazine, Vol. 14, No. 2, pp. 6-17, 1998.

[2] A. Beroual and R. Tobazeon, "Pre-breakdown Phenomena in Liquid Dielectrics", IEEE Trans. Electr. Insul., Vol. 21, pp. 613-627, 1986.

[3] A. Beroual, "Electronic and Gaseous Processes in Prebreakdown Phenomena of Dielectric Liquids", J. Appl. Phys. Vol. 73, pp. 45284533, 1993.

[4] T. Aka-Ngnui and A. Beroual, "Modeling of Multi-Channel Streamers Propagation in Liquid Dielectrics using the Computation Electrical Network", J. Phys. D: Appl. Phys. Vol. 34, pp.794-805, 2001.

[5] T. Aka-Ngnui and A. Beroual, "Modeling of branching streamers propagating within dielectric liquids", IEEE Conf. Electr. Insul. Dielectr. Phenomena (CEIDP), Ontario, Canada, pp.448-451, 2001.

[6] I. Fofana and A. Beroual, "Predischarge Models in Dielectric Liquids", Jpn. J. Appl. Phys., Vol. 37, pp. 2540-2547, 1998.

[7] I. Adamczewski, "Les Phénomènes d'Ionisation et de Conduction dans les Diélectriques Liquides", Adaptation Française, MASSON et $\mathrm{C}^{\mathrm{ie}}$, EDITEURS, 1968.

[8] I. Fofana and A. Beroual, "Predischarge Models in Dielectric Liquids", Jpn. J. Appl. Phys. Vol. 37, pp.2540-2547, 1998.

[9] K. L. Stricklett, C. Fenimore, E.F. Kelley, H. Yamashita, M. O. Pace, T. V. Blalock, A. L. Wintenberg and I. Alexeff, "Observation of Partial Discharge in Hexane under High Magnification", IEEE Trans. Electr. Insul., Vol. 26, pp. 692-697, 1991.
[10] R. Badent, K. Kist and A. J. Schwab, "Investigation of Pre-breakdown Phenomena in Insulating oil", 8th ISH, Yokohama (Japan), pp. 24-03, 1993.

[11] A. Beroual and T. Aka-Ngnui, "Influence of Additives and Hydrostatic Pressure on Streamers Initiation and Dielectric Strength of Liquids", IEEE Conf. Electr. Insul. Dielectr. Phenomena (CEIDP), Cancun, Mexico, pp.248-251, 2002.

[12] T. J. Gallagher, Simple Dielectric Liquids : Mobility, Conduction and Breakdown, Oxford University Press, 1975.

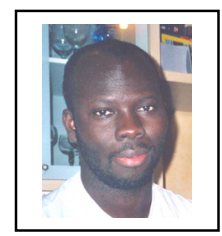

Thomas Aka-Ngnui was born in Abidjan, Côte d'Ivoire in 1970 . He received his physical engineering degree from the Faculty of Science and Technology, University of Abidjan, Côte d'Ivoire in 1995, the M.Sc. degree and the Ph.D. degree from the Ecole Centrale, Lyon, France in 1996 and 2000, respectively. He is presently an associate professor at the Ecole Centrale de Lyon where he works in the Dielectric Materials and High Voltage team at the Centre de Génie Electrique de Lyon (CEGELY) CNRS. His main research interests include high voltage phenomena, electrical insulation, dielectric materials, modeling and computer simulation. $\mathrm{He}$ is author/co-author of more than 10 papers.

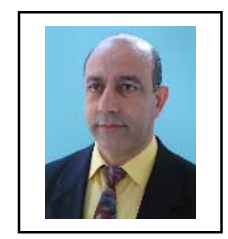

Abderrahmane Beroual (M'92) was born on 30 October 1951. He received the Dipl. Ing. and the Ph.D. degrees in electrical engineering, respectively in 1976 from the Ecole Nationale Polytechnique of Algiers, Algeria and in 1979 from the Technical University of Wroclaw, Poland. In 1987, he obtained the Doctorat d'Etat ès Sciences Physiques degree from the Institut National Polytechnique of Grenoble, France. In 1980, he joined the Department of Electrical Engineering at the Ecole Nationale Polytechnique of Algiers. From 1983 to 1987 , he was a researcher at the Laboratoire d'Electrostatique et de Matériaux Diélectriques (LEMD) - CNRS of Grenoble, France and associate professor at the Joseph Fourier University of Grenoble. He received the title of professor at the Ecole Nationale Polytechnique of Algiers in 1987. In 1989, he joined the Ecole Centrale de Lyon, France where he is presently professor and the head of the Dielectric Materials and High Voltage team at the Centre de Génie Electrique de Lyon (CEGELY) - CNRS as well as responsible for the Master Research Program in Electrical Engineering. He is responsible for numerous research projects. His main research interests include high voltage insulating, dielectric materials, long air discharge and lightning, modelling of discharges and composites. He is author/co-author of more than 230 papers and four patents. $\mathrm{He}$ supervised about $30 \mathrm{Ph} . \mathrm{D}$. theses. He was the leadership of the International Study Group on Streamer Propagation in Liquids of the IEEE DEIS (1994 - 1998). He is on the International Advisory Committees of the International Conference on Dielectric Liquids and the International Conference on Advances in Processing, Testing and Application of Dielectric Materials. 